\title{
AN ARCHITECTURE OF DEEP LEARNING METHOD TO PREDICT TRAFFIC FLOW IN BIG DATA
}

\author{
Leelavathi M V ${ }^{1}$, Sahana Devi K $\mathbf{J}^{\mathbf{2}}$ \\ ${ }^{I}$ M. Tech SCS, Department Of Computer Science \& Engineering, East West Institute Of Technology, Bangalore. \\ Karnataka, India \\ leela.monst@gmail.com \\ ${ }^{2}$ Assistant Professor, Department Of Computer Science \& Engineering, East West Institute Of Technology, Bangalore, \\ Karnataka, India \\ sahanadevi5@gmail.com
}

\begin{abstract}
The invent of IEEE 802.11p as a communication standard, specific network protocol called vehicular adhoc network (VANET) based on mobile adhoc network ( MANET) along with sensor technology has put a strong foundation to visualize as well as make a reality of various intelligent transport applications \& systems (ITAS) for safety and comfort. The success of such conceptualized applications depends upon how precisely as well reliability (in term of timeliness) the "traffic flow prediction" is done. The constitute of traffic data is characterized as Big Data type, therefore existing traffic prediction models are not in a capacity to provide the accurate result for various ITAS as the existing models consider low traffic data which lacks the insight of Big Data. In order to overcome these limitations, the synopsis aims to solve the "traffic flow prediction problem" by a novel mechanism of rigorous-learning based prediction model (RLBPM) using big traffic data. The RLBPM will exploit the spatial-temporal correlation statistics and for the purpose of learning algorithm "stacked auto encoder (SAE) model" will be used. The outcome of the RLBPM is expected to perform more superior as compared to the existing traffic flow prediction model. The implementation strategy involved mathematical modeling and simulation using Matlab.
\end{abstract}

Keywords: VANET, MANET, ITAS, Big Data, SAEs, RBNN, GRNN.

\section{INTRODUCTION}

In the recent times achieving an accuracy associated with the vehicular traffic information has become a crucial challenge in the field of vehicular ad hoc communications as broadcasting and prediction of traffic information is strongly recommended for the individual travelers of a big metro city where the people wants to attend their office within an estimated time period. The main objective associated with the prediction of traffic information is to provide the traffic information to each and every vehicle associated with one network in order to optimize the traffic congestion issues in vehicular adhoc networks (VANET) [1] [2]. The recent conventional studies highlights that now a days the deployment of Intelligent Transport Systems (ITSs) has improved the traffic operation efficiency with respect to better travel decision making, reducing carbon emissions optimization of level of congestion. The traffic flow congestion prediction has been conceptualized considering a huge set of historical and traffic arranged information gathered from different sensor sources. There can be such a large number of different sensor sources, for example, inductive circles, radars, cameras and so forth.

As each and every sensor node generates a huge amount of data that is how the generation of huge traffic data is exploding therefore, sensor technology has become somehow connected with the generation of big data in transportation systems. However transportation system now a days has become more data driven and thus many conventional traffic flow prediction and congestion control mechanisms have been introduced where most of them have been found unsatisfactory [3][4]. Now a days the deep learning methodology which utilizes the concept of machine learning so as to concentrate valuable components in information from the lower level to the most abnormal amount.

Deep learning model has been designed considering a multilevel architecture which eases the computation of normal language preparing, information dimensionality lessening and object identification. It can be seen that as the traffic flow control models are considered as very complicated architectures thus deep learning can be used to represent the traffic features without the prior knowledge.

The proposed project aims to introduce a neural network and deep learning based model with a specific end goal to anticipate the movement stream in the field of big data and analytics. The following Figure. 1 shows a block diagram of an artificial neural network model [5]. 


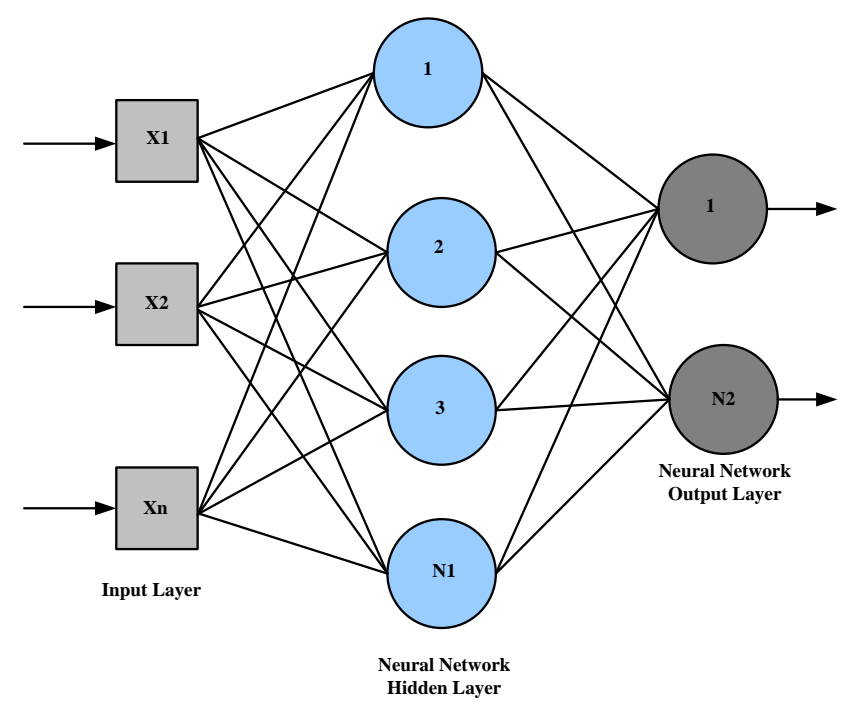

Figure 1: Block Diagram of an Artificial Neural Network System.

The above figure. 1 also shows that there are three different types of layers are associated with neural network models which are 1. Input layer 2. Neural Network Hidden layer 3. Output layer. The input layer uses a set of traffic oriented data set to train the hidden layer of the neural network which is further used to anticipate the movement stream in a specific zone.

The output layer shows the different predicted values after performing the training of the neural network. In this paper we proposed An Architecture of Deep Learning method to Predict Traffic flow in big data. The paper is sorted out as takes after Section II examines about the late studies towards activity stream forecast utilizing profound learning technique. Section III discusses about proposed system and implementation part. Section IV discusses about the result and discussion and analysis followed by conclusion in Section V.

\section{LITERATURE REVIEW}

This section deals with the studies of Big data (BD) over deep learning (DL). There are many researchers are contributed in $\mathrm{BD}$ but less in number for $\mathrm{DL}$ and are discussed as follows.

Leung et al. [1] have discussed the introduction of machine learning (ML) tasks, to address the issues in genomic medicine (GM) (helps in determination of individual DNA variations). The study provides the platform for future computational method for effective GM.

Zhang and Chen [2] has presented a paradigm of distributed learning for Restricted Boltzmann Machines (RBMs) and also an algorithm of a back propagation (BP) by using Map Reduce and a programming model parallel. The author has gone through the Deep Belief Nets (DBNs) and RBNs. The DBN learning starts with the RBNs series restraining and by fine tuning of entire net by using BP. Basically, the implementation of both BP algorithm and the RBMs will provide useful computation time to process the huge data sets. The authors have validated the various problems of data sets and outcome with the results of better performance as efficiency and accuracy point of view by distributed DBNs and RBNs.

Chen and Lin [3] has given an overview of deep learning (DL), past research efforts and also the challenges of big data and provided a future scope for DL in machine learning (ML). As per the study of the author, DL is the active topic in the research field and has got popularity due its vast application in many areas like speech recognition, language processing and also provides solutions in Big Data Analytics (BDA).

Wu et al. [4] have presented a weekly semi-supervised deep learning for multi-label image annotation (WeSed) approach in deep learning. The author has studied both weakly labeled and unlabeled type of images for annotation of multi-label image. The experiments on these types of images result from better performance by using the proposed approach.

Zhang et al. [5] have illustrated a framework to obtain the underlying circumstances for big video data, the framework comprised of an approach which combines both historical and current view to have better context in which DL techniques are used to obtain the raw context data. Authors have carried out initial evaluation to represent the proposed approach effectiveness and accuracy in obtaining the raw contexts. The approach outcomes with effective results in a real time context awareness in video cloud.

Zhou et al. [6] have presented Stacked Extreme Learning Machine (S-ELMs) to solve the issues of large and complex data. Authors have studied past researches on ELMs. The proposed S-ELMs divide the large network of ELM into some stacked ELMs, which connected in series. The SELMs helps in approximation of large ELM system, where the small memory is required. Study outcomes with a better accuracy in testing (than Support Vector Machine) and slightly better accuracy than DBN with faster speed in training.

Yisheng et al. [7] have presented a traffic flow prediction (TFP) method based on deep learning. Authors have worked on the increasing traffic data issues in real-time applications. A stacked autoencoder ( $\mathrm{S}-\mathrm{AE}$ ) model is utilized to take in the insipid elements of movement stream. The experiment outcomes with the superior performance based TFP method.

Park et al. [8] have presented the low-cost platform for DL applications like mobile and other portable devices. Author have worked on DL algorithm and implemented energy 
efficient DL and interference processors for wearable systems. Study outcomes with the energy efficient model than the state of art.

Jun Wang et al. [9] have discussed the survey of learning to hash framework, various types of representative techniques, composed of supervised, semi supervised and unsupervised. The study presents future research flow requirement in BDA.

Zhang et al. [10] have illustrated a deep computational model to learn the big data features. The model uses the tensor distance in the output layer as the average sum of squares error term of reconstruction error. The model is experimented on four datasets by comparing them with multi model DL model and stacking auto encoders. The study results illustrate that the model is efficient in learning using CUAVE, INEX, SANE and STL-10 datasets.

Huang et al. [11] proposed a Traffic stream figure is used here for unsupervised component learning. It can learn fruitful parts for action stream desire in an unsupervised way, which has been examined and seen to constrain for a few districts, for instance, picture and sound order; we propose a gathering strategy in light of the weights in the top layer to make MTL more successful. These positive results exhibit that profound learning and MTL are promising in transportation research.

Hinton et al. [12] introduced a novel profound learningbased movement stream expectation strategy. This considers the spatial and common connections intrinsically. A stacked auto encoder model is used to learn non-particular movement stream segments, and it is set up in an insatiable layer sagacious configuration. Here a profound design model is connected utilizing auto encoders as building squares to speak to activity stream highlights for expectation. Besides, tests show that the proposed strategy for activity stream expectation has unrivaled execution.

Chang et al. [13] displayed an element multi interim movement volume expectation model in light of the k-NN nonparametric relapse. The improvement of a piece smoother for the auto relapse capacity to do fleeting movement stream forecast, in which useful estimation procedures were connected.

Tan et al. [14] proposed an aggregate procedure for development stream estimate in perspective of the moving ordinary (MA), exponential smoothing (ES), ARIMA, and neural framework (NN) models. The MA, ES, and ARIMA models were used to gain three huge time course of action that were the reason of the $\mathrm{NN}$ in the amassing stage. Zargari et al. [7] made particular straight innate programming, multilayer perceptron, and soft method of reasoning (FL) models for assessing 5-min and 30-min movement stream rates.

Zang et al. [15] explores the investigation of the spatialtransient data of cell movement stream and the forecast of cell-station activity volumes. Taking into account the joining of K-means bunching, Elman Neural Network and wavelet disintegration techniques, we portray the execution examination of movement volume expectation. The assessment of our wavelet decay based machine learning approach utilizing the genuine movement information recorded at a locale in a major city and exhibited the increase over conventional methodologies.

Urushibara al. [16] done examination investigations of movement stream expectation models have been accounted for in writing. The straight relapse, the authentic normal, the ARIMA, and the SARIMA were surveyed in his paper and in which it was inferred that these calculations perform sensibly well amid ordinary working conditions yet don't react well to outside framework changes. The SARIMA models and the nonparametric relapse estimating techniques were assessed in.

\section{PROPOSED METHOD IMPLEMENTATION}

We proposed architecture of deep learning method to predict the traffic flow in big data framework. The exact and opportune activity stream data is vital for the effective arrangement of smart transportation frameworks. Throughout the most recent couple of years, movement information has been blasting, and what's more, we have really entered the time of tremendous data for transportation. Existing movement stream expectation techniques predominantly utilize shallow activity forecast models are as yet unsuitable for some certifiable applications. These circumstances rouse us to reevaluate the movement stream expectation issue in view of profound design models with enormous activity information. Figure. 2 demonstrates the general framework design of the proposed technique.

The auto encoder is a special type of neural network (NN), where the objective yield turns into the contribution for the model. A neural system comprising of one's of every info layer, shrouded layer and yield layer each is appeared in the auto encoder design. The training samples are represented by a set of $T s=\{x(1), x(2), x(3) \ldots x(i)$, Where, $x(i) €$ $\mathrm{Rd}$, The sample data will be generated synthetically by developing some random/ pattern based sample generation function. The training and test datasets are segregated accordingly. With these given set of sample data, the process flow of the auto-encoder for its encoding and decoding process is described into the process flow diagram shown in figure 2 . 


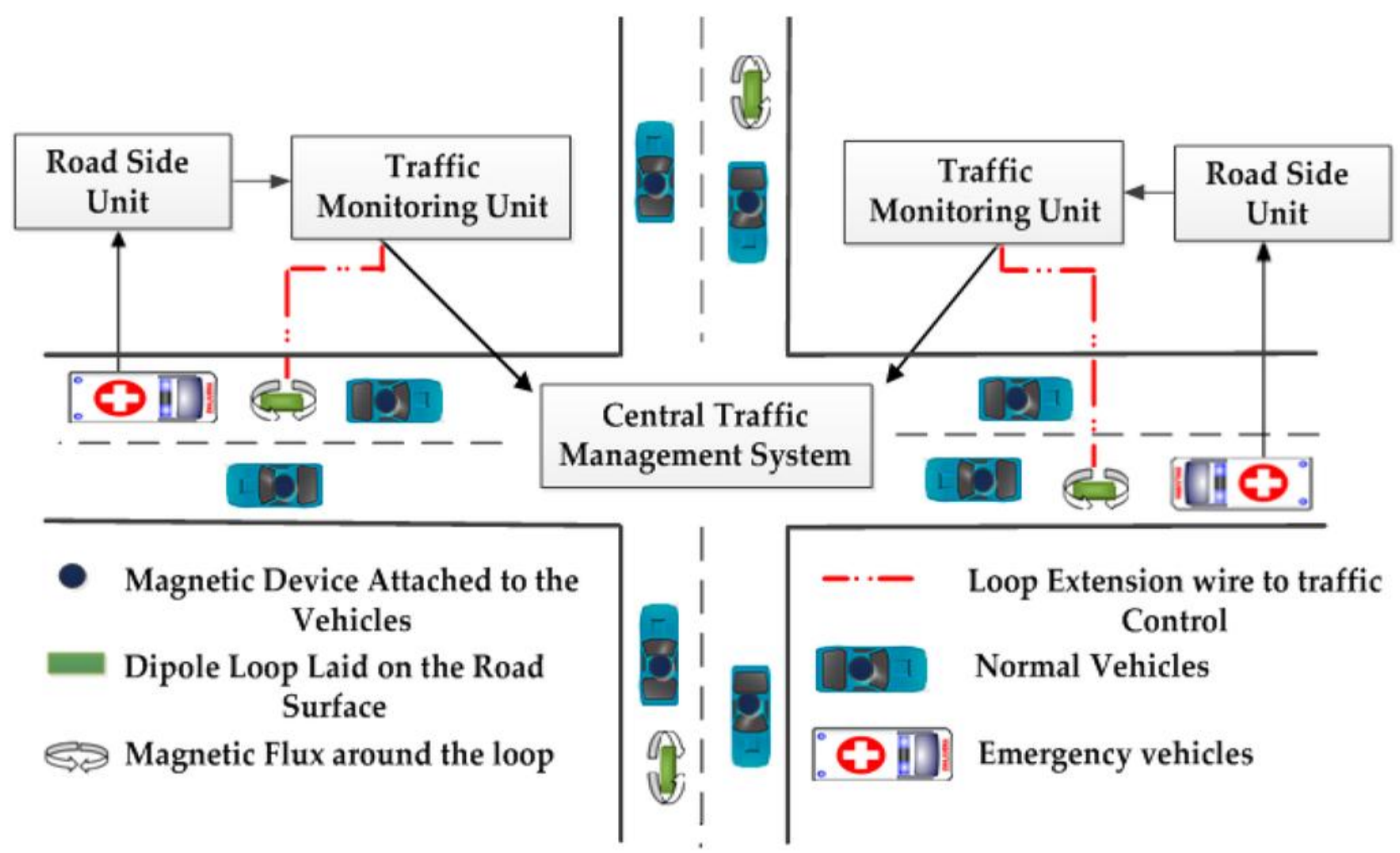

Figure 2: General System Architecture of the Proposed Model

The objective of this module namely "stacking of AEs" is to form a deep network by means of stacking auto encoders in a particular fashion as described below: The output of one autoencoder will become the input for the next layer of the stack. The very first hidden layer is trained by the actual input data and then it acts as input contribution to the second shrouded layer. Summarily, the yield of the nth shrouded layer will turn into the contribution for the $n+1$ th concealed layer.

Table.1: Symbols used in the Mathematical Model

\begin{tabular}{|c|c|c|}
\hline Sl. No & Symbol & Description \\
\hline 1 & $\mathrm{~W}_{1}$ & Weight matrix \\
\hline 2 & $\mathrm{~B}$ & Encoding bias vector \\
\hline 3 & $\mathrm{~W} 2$ & Decoding matrix \\
\hline 4 & $\mathrm{C}$ & Decoding bias vector \\
\hline 5 & $\mathrm{~T}_{\mathrm{s}}$ & Training Sample \\
\hline 6 & $\mathrm{X}^{\mathrm{i}}$ & $\mathrm{i}^{\text {th }}$ Training Sample \\
\hline 7 & $\mathrm{R}^{\mathrm{d}}$ & Real data \\
\hline
\end{tabular}

The hierarchical organization of the autoencoders is presented in figure.3. A predictor is needed as the top layer for supervised learning; a logistic regression layer is used as predictor in the current work. Collectively all the layers together with the predictor form the deep network.

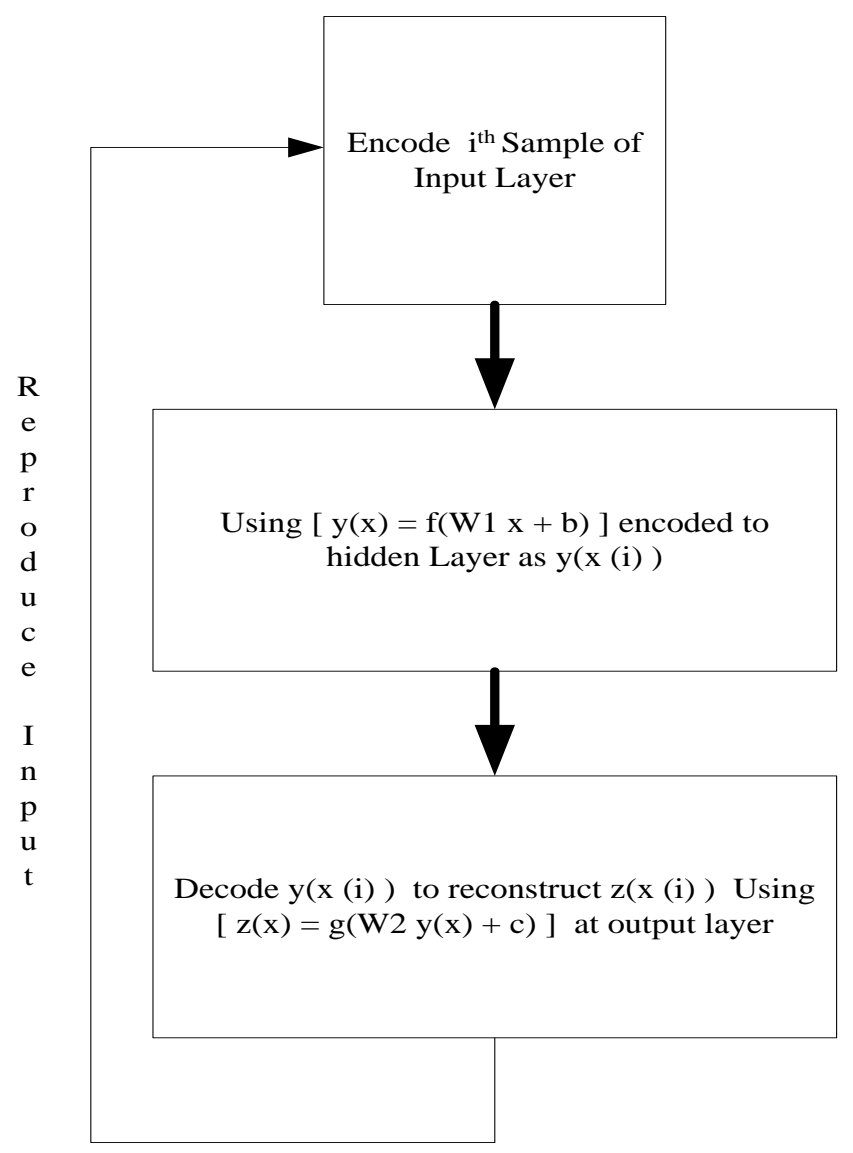

Figure.3: Process flow diagram of the Auto encoder 


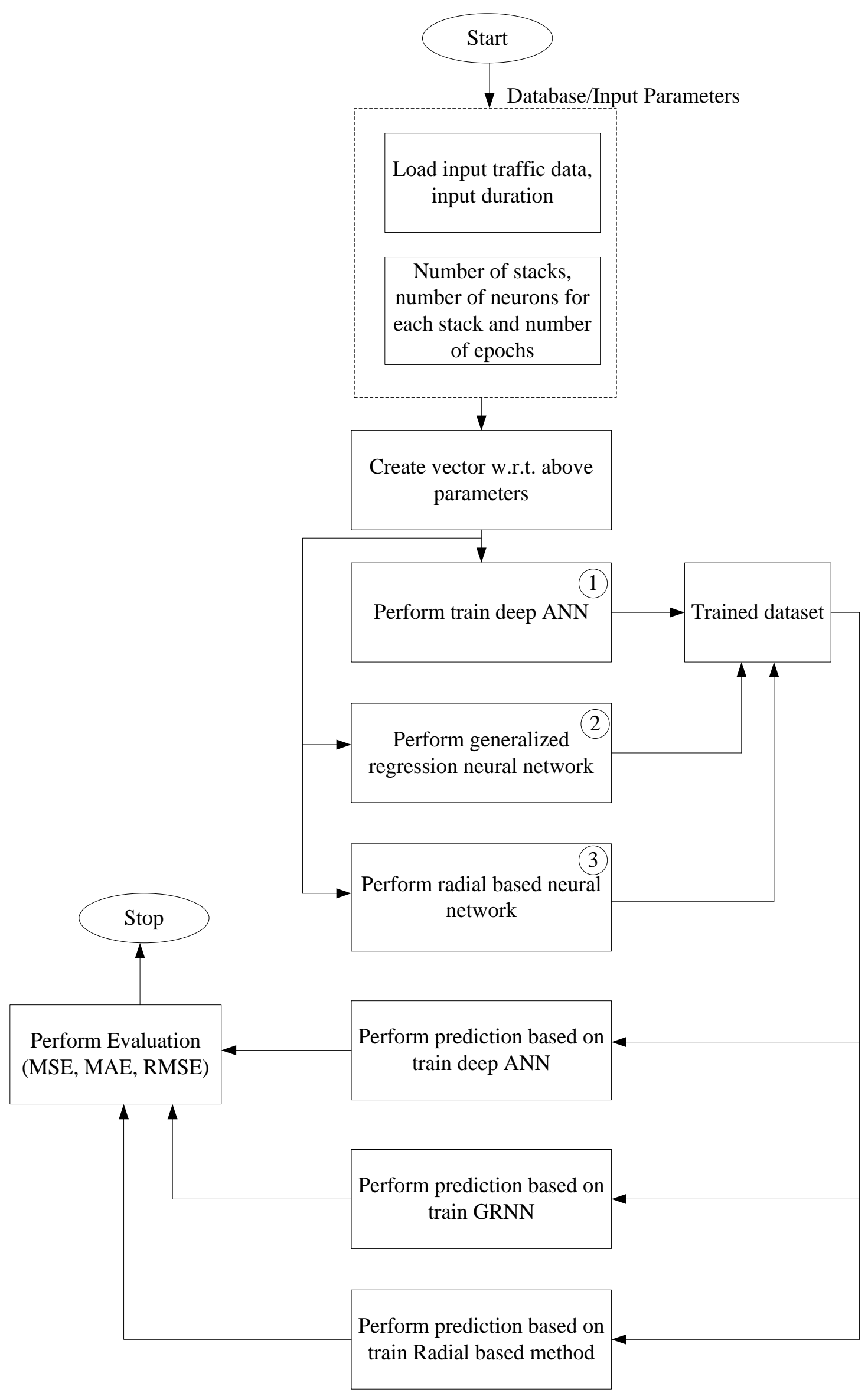

Figure 4: Process Flow Diagram of proposed method 
Exclusively using the back propagation along with the gradient-descent based optimization is proved to be slow and sometimes halts in deep networks, as noted by James Martens et al. [1]. Hence greedy layer wise unsupervised algorithm is considered for training, as suggested by Hinton et al. [2], to train the network starting from hidden layer 1. Finally, back propagation is only used to fine-tune the network from the inverse side of shrouded layers and proceed till the principal concealed layer. Steps to train the network using greedy layer-wise approach:

- Training should be done in first layer using autoencoder model and set as input for the second hidden layer.

- The second step is training should be done using next layer using the output of first layer is given to the input to the second layers.

- Repeat step 2 for all the hidden layers

- Initialize prediction layer randomly.

- Train the prediction layer by using the last hidden layer as input

Steps to fine-tune the network using back propagation:

- Initialize the weight matrix, and bias vector randomly

- Use the gradient-based optimization technique for tuning the network parameters; start from the prediction layer, last hidden layer, and proceed till the first hidden layer.

\section{RESULTS AND DISCUSSION}

This section gives the result obtained in this proposed method. Here, we implemented the deep learning architecture model to predict the traffic flow. Here, firstly we created a database as a numerical case. The traffic flow data are gathered for each $15 \mathrm{~min}, 30 \mathrm{~min}, 45 \mathrm{~min}$ and $60 \mathrm{~min}$ separately. The gathered data are totaled $5 \mathrm{~min}$ interim each for every identifier station. In this work, the activity stream information are utilized for the reenactment purposes. The information of the initial two months were chosen as the preparation set, and the staying one month's information were chosen as the testing set. For turnpikes with various indicators, the activity information gathered by various finders are accumulated to get the normal movement stream of this interstate. Note that we independently treat two bearings of the same interstate among all the turnpikes, in which three are one-way. Figure.5 is a plot of a commonplace turnpike's activity stream after some time of $5 \mathrm{~min}$.

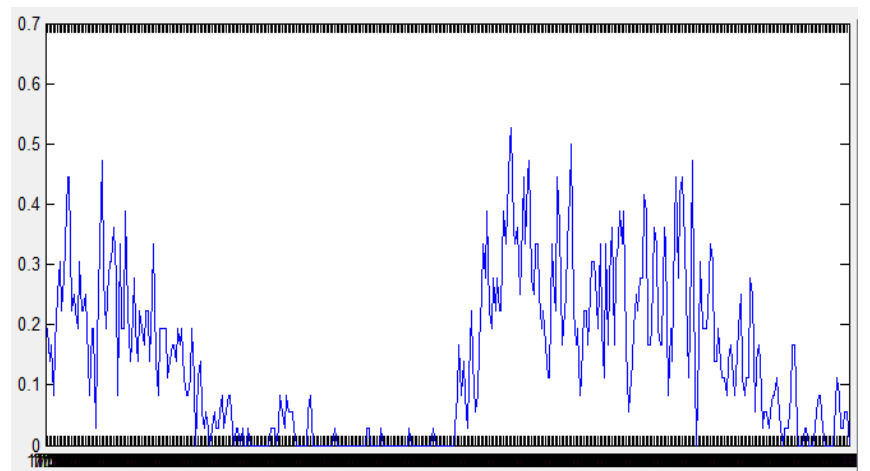

Figure 5: Plot of traffic data for $5 \mathrm{~min}$
In this simulation work we choose a number of stacks as 1 . That is the number of layers as only one. Then the stack size is 100. The number of epochs are 100 are selected. After this applying the Deep NN method (SAE method) to train the traffic data and collects all the features present in this data. Then, store all these feature in a database.

In this method also we chooses a number of stacks as 1 . That is the number of layers is one. Then the stack size is 100. The number of epochs are 100 are selected. After this applying the General Regression Neural Network method (GRNN method) to train the traffic data and collects all the feature present in this data. Then, store all these feature in a database. Figure. 6 shows the training model for traffic data using generalized regression neural network.

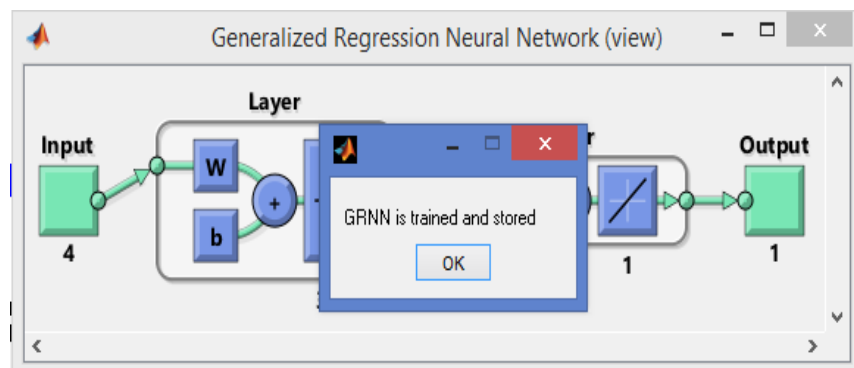

Figure 6: Training of traffic data using GRNN method

In RBNN method, we choose a number of stacks as 1 . That is the number of layers is one. Then the stack size is 100 . The number of epochs are 100 are selected. After this applying the Radial Basis Neural Network (RBNN method) to train the traffic data and collects all the features present in this data. Then, stores all these feature in a database. Figure.7 shows the training model for traffic data using radial basis neural network method.

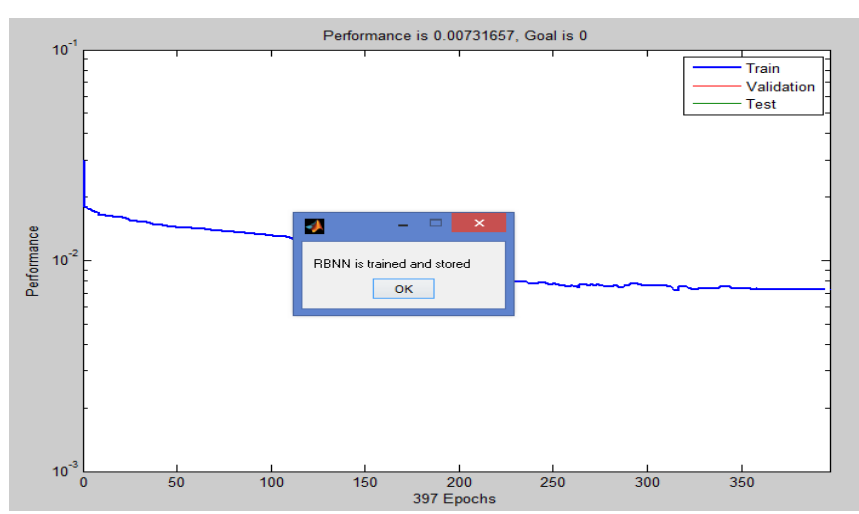

Figure 7: Training of traffic data using RBNN method 


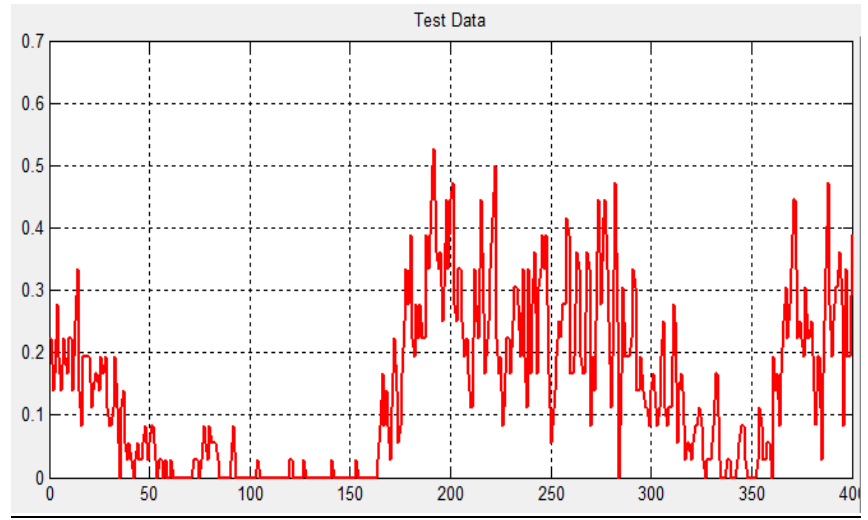

Figure 8: Test data

With respect to the structure of a SAE system, we have to decide the span of the information layer, the quantity of shrouded layers, and the quantity of concealed units in each shrouded layer. For the information layer, we utilize the information gathered from all interstates as the information; in this manner, the model can be worked from the point of view of a transportation system considering the spatial relationships of movement stream.

Figure. 9 shows the predicted traffic data using the proposed method. Here, we can observe that, our proposed method almost matches the original data. So, it predicts the data almost equal to original data. The evaluation is done the assessment is done viably by the proposed model; we utilize three execution lists, which are the MAE, MRE and RMSE. The MAE obtained is 0.076857 , MRE obtained is 0.80111 and RMSE obtained is 0.091958 .

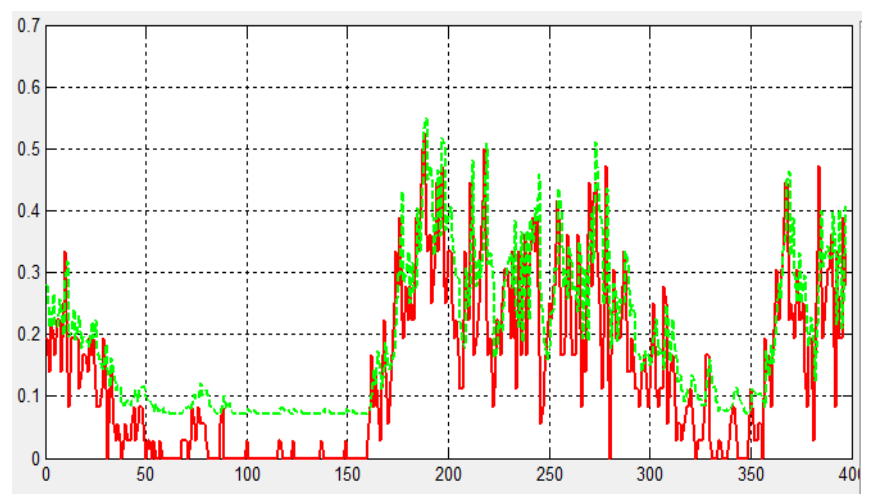

Figure 9: Predicted traffic data using proposed method

Figure 10 shows the predicted traffic data using the GRNN method. Here, we can observe that, this method gives less accuracy compare to our proposed method. For the evaluation purpose we used the following three parameters are: which are MAE, MRE and RMSE. The MAE obtained is 1.10592, MRE obtained is 0.896 and RMSE obtained is 0.12573 .

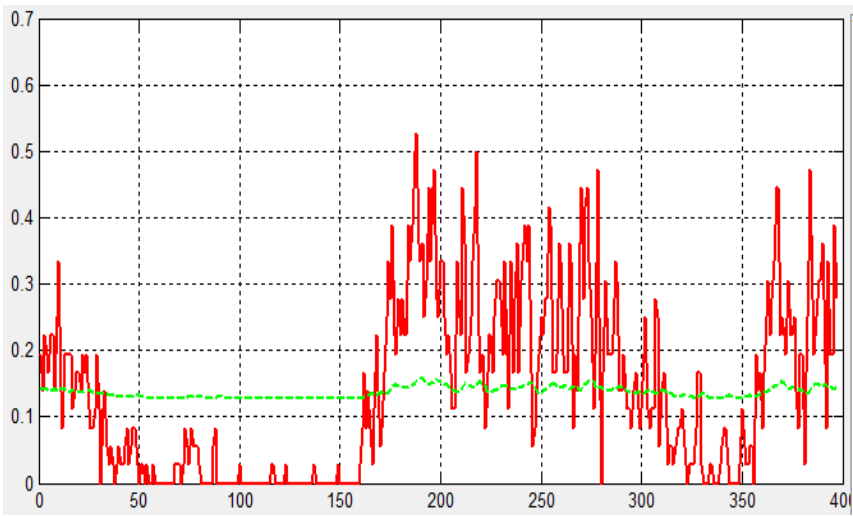

Figure 10: Predicted traffic data using GRNN method

Figure.11 shows the predicted traffic data using the RBNN method. Here, we can observe that, this method gives less accuracy compare to our proposed method. For the evaluation purpose we used the following three parameters are: which are the MAE, MRE and the RMS error. The MAE obtained is 0.083075 , MRE obtained is 0.87287 and RMSE obtained is 0.24298 .

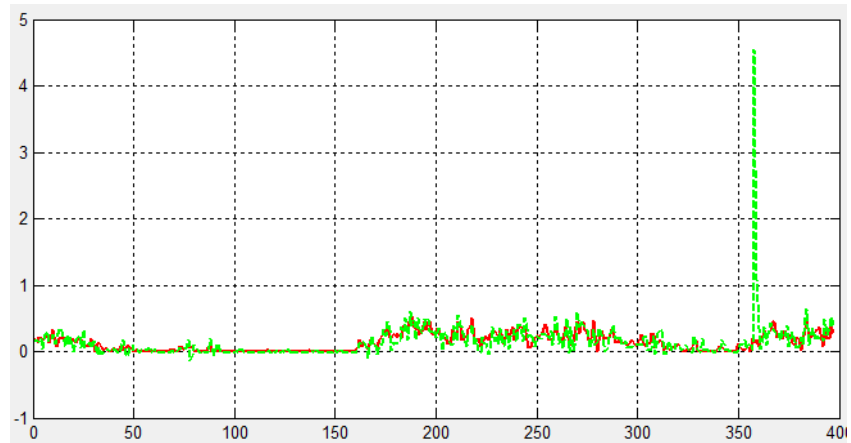

Figure 11: Predicted traffic data using RBNN method

We assessed the execution of the proposed technique on a dataset and contrasted it and the SAEs (Proposed) Method, the RBSS and the GRNN strategies, and the outcomes demonstrates that the proposed strategy is better than the contending strategies. Figure.12 demonstrates the examination of the two distinct strategies with the proposed methods.

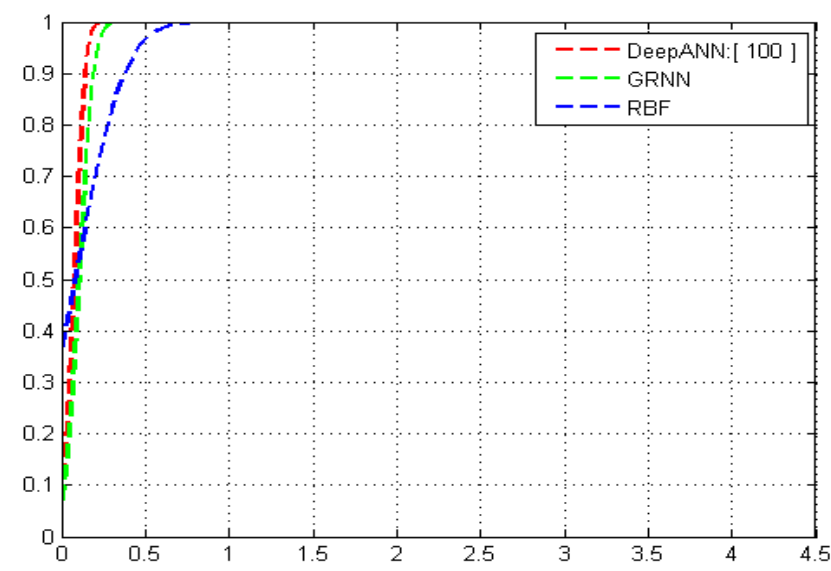

Figure 12: Comparison of two different methods with the proposed method. 
We assessed the execution of the proposed technique on a dataset and contrasted it and the SAEs (Proposed) Method, the RBSS and the GRNN strategies, and the outcomes demonstrates that the proposed strategy is better than the contending strategies. Figure.12 demonstrates the examination of the two distinct strategies with the proposed methods.

\section{CONCLUSION AND FUTURE DIRECTION}

The recent advancements in vehicular traffic congestion control has brought the concept of advance traffic flow prediction using neural network tool therefore designing an efficient traffic congestion prediction and control somehow minimized the congestion and people can reach their destination safely within an estimated period of time. This project has introduced an efficient Artificial Neural Network based traffic flow control mechanism which uses the previous year's traffic oriented big datasets in order to predict the traffic flow. Training of the neural network is performed for accurate prediction of traffic flow and congestion within a particular area. The performance analysis of the proposed system has been evaluated using an experimental prototyping.

\section{REFERENCES}

[1] Leung, G. M., W. W. Lim, L-M. Ho, T-H. Lam, A. C. Ghani, C. A. Donnelly, C. Fraser et al. "Seroprevalence of IgG antibodies to SARS-coronavirus in asymptomatic or subclinical population groups." Epidemiology and infection 134, no. 02 (2006): 211221.

[2] Zhang, Jie, Shiguang Shan, Meina Kan, and Xilin Chen. "Coarse-to-fine auto-encoder networks (cfan) for realtime face alignment." In Computer Vision-ECCV 2014, pp. 1-16. Springer International Publishing, 2014.

[3] Chen, X.W. and Lin, X., 2014. Big data deep learning: challenges and perspectives. Access, IEEE, 2, pp.514525.

[4] Wu, F., Wang, Z., Zhang, Z., Yang, Y., Luo, J., Zhu, W. and Zhuang, Y., 2015. Weakly Semi-Supervised Deep Learning for Multi-Label Image Annotation. Big Data, IEEE Transactions on, 1(3), pp.109-122.

[5] Zhang G, Lu J, Wu D, Mao M, Wang W, . Recommender system application developments: a survey. Decision Support Systems. 2015 Jun 30;74:1232.

[6] Zhang, Z., Wu, F., Wang, Z., , Yang, Y., Luo, J., Zhu, W. and Zhuang, Y., 2015. Weakly Semi-Supervised Deep Learning for Multi-Label Image Annotation. Big Data, IEEE Transactions on, 1(3), pp.109-122.

[7] Yisheng, S.H.A.O., 2004. Control and plan of city water system. City Planning Review, 10, pp.63-67.

[8] [8]Park G,Mascarenas DL, Todd MD, Farrar CR. Development of an impedance-based wireless sensor node for structural health monitoring. Smart Materials and Structures. 2007 Oct 8;16(6):2137.

[9] [9]Wang, G.,Xia, J., Lv, A. and Tan, G., 2003. A research on distributed time variant gain modeling.
ACTA GEOGRAPHICA

EDITION-, 58(5), pp.789-796.

[10]Zhang, Q., Yang, L.T. and Chen, Z., Deep Computation Model for Unsupervised Feature Learning on Big Data, Retrived, 19th April, 2016

[11]Huang, W., Song, G., Hong, H. and Xie, K., 2014. Deep architecture for traffic flow prediction: deep belief networks with multitask learning. Intelligent Transportation Systems, IEEE Transactions on, 15(5), pp.2191-2201.

[12] Hinton, Geoffrey E., Simon Osindero, and Yee-Whye Teh. "A fast learning algorithm for deep belief nets." Neural computation 18, no. 7 (2006): 1527-1554.

[13] Chang, H., Youngjoo Lee, Byung-Jun Yoon, and Sanghoon Baek. "Dynamic near-term traffic flow prediction: systemoriented approach based on past experiences." Intelligent Transport Systems, IET 6, no. 3 (2012): 292-305.

[14]Tan, M.C., Wong, S.C., Xu, J.M., Guan, Z.R. and Zhang, P., 2009. An aggregation approach to shortterm traffic flow prediction. Intelligent Transportation Systems, IEEE Transactions on, 10(1), pp.60-69.

[15]Zang, Y., Ni, F., Feng, Z., Cui, S. and Ding, Z., 2015, July. Wavelet transform processing for cellular traffic prediction in machine learning networks. In Signal and Information Processing (ChinaSIP), 2015 IEEE China Summit and International Conference on (pp. 458-462). IEEE.

[16] Urushibara, A., Moritomo, Y., Arima, T., Asamitsu, A., Kido, G. and Tokura, Y., 1995. Insulator-metal transition and giant magnetoresistance in La 1- $\mathrm{x}$ Sr $\mathrm{x}$ MnO 3. Physical Review B, 51(20), p.14103.

[17] Stathopoulos, A. and Karlaftis, M.G., 2003. A multivariate state space approach for urban traffic flow modeling and prediction. Transportation Research Part C: Emerging Technologies, 11(2), pp.121-135.

[18]Lippi M, Bertini M, Frasconi P. Short-term traffic flow forecasting: An experimental comparison of time-series analysis and supervised learning. Intelligent Transportation Systems, IEEE Transactions on. 2013 Jun;14(2):871-82.

[19] Chen, C., Wang, Y., Li, L., Hu, J. and Zhang, Z., 2012. The retrieval of intra-day trend and its influence on traffic prediction. Transportation research part $\mathrm{C}$ : emerging technologies, 22, pp.103-118. 